\title{
Near Infrared Survey of the Nuclear Regions of the Milky Way
}

\author{
U. C. Joshi ${ }^{1}$, S. Ganesh ${ }^{1}$, K. S. Baliyan ${ }^{1}$, I. S. Glass ${ }^{2}$, and T. Nagata ${ }^{3}$ \\ ${ }^{1}$ Physical Research Laboratory, India \\ ${ }^{2}$ South African Astronomical Observatory, South Africa \\ ${ }^{3}$ Nagoya University, Japan
}

\begin{abstract}
Results based on the deep imaging survey of the inner region ( $\sim 300 p c$ of the bulge within $|\ell| \sim 1.5^{\circ},|b| \sim 0.5^{\circ}$ ) of the Milky Way are reported in this communication. This survey is about 2.5 magnitude deeper than DENIS and 2MASS and is able to detect stars of the red clump at a distance of the Galactic center. Toward some directions we find extinction reaching $A_{V}=50$ mag. A catalog of the sources is in preparation.
\end{abstract}

Keywords. Milky-way, interstellar extinction, Galactic Bulge

\section{Introduction}

In the recent past there have been several NIR surveys, e.g.DENIS, 2MASS. Due to poor spatial resolution, these surveys suffer from confusion and hence lack depth in the high number density nuclear region of the Galaxy. The interstellar extinction for the inner Galactic Bulge is detected to be $>25 \mathrm{mag}$ with a clumpy, inhomogeneous nature(Schultheis et al., 1999 A\&A, 349, L69). A large number of $K_{S}$ sources do not have counterparts at shorter wavebands in DENIS and 2MASS. To overcome these problems, and to gain a better understanding of the distribution of stellar populations in the nuclear bulge region, we carried out deep imaging survey of the inner region in $J, H \& K_{S}$ bands using IRSF telescope, SAAO, Sutherland, during June-July 2002 (for details see Baliyan et al. 2003 ANS, 324, p47).

\section{Results}

We use 0.1sec exposure data to plot CMDs: $J-K_{S}$ vs $K_{S}$ (fig 1 ) and $H-K_{S}$ vs $K_{S}$ (fig 2) for 9 locations around $(\mathrm{RA}=17: 48, \mathrm{DEC}=-28: 20)$. All the figures are in the online section. Note that the number of sources detected in both $H$ and $K_{S}$ is much larger than that detected also at $J$. In fig $3\left(H-K_{S}\right.$ vs $K_{S}$ for GC1748-2820F) we have also shown the red giant isochrones at different $A_{V}$ ranging from 30 to 50 mag. With the longer integration data we estimate and quantify accurately the extinction to much deeper values. Final products - a Catalog of $\sim 3$ million sources with extinction and stellar population identification and an extinction map towards the Nuclear Bulge, will be published shortly.

\section{Acknowledgements}

This work was supported by the Department of Space, Govt. of India. We are thankful to the SAAO for providing telescope time. We(SG \& KSB) also acknowledge the SAAO for the support during our observing run at Sutherland and stay at Cape Town. 


\section{Online Section}
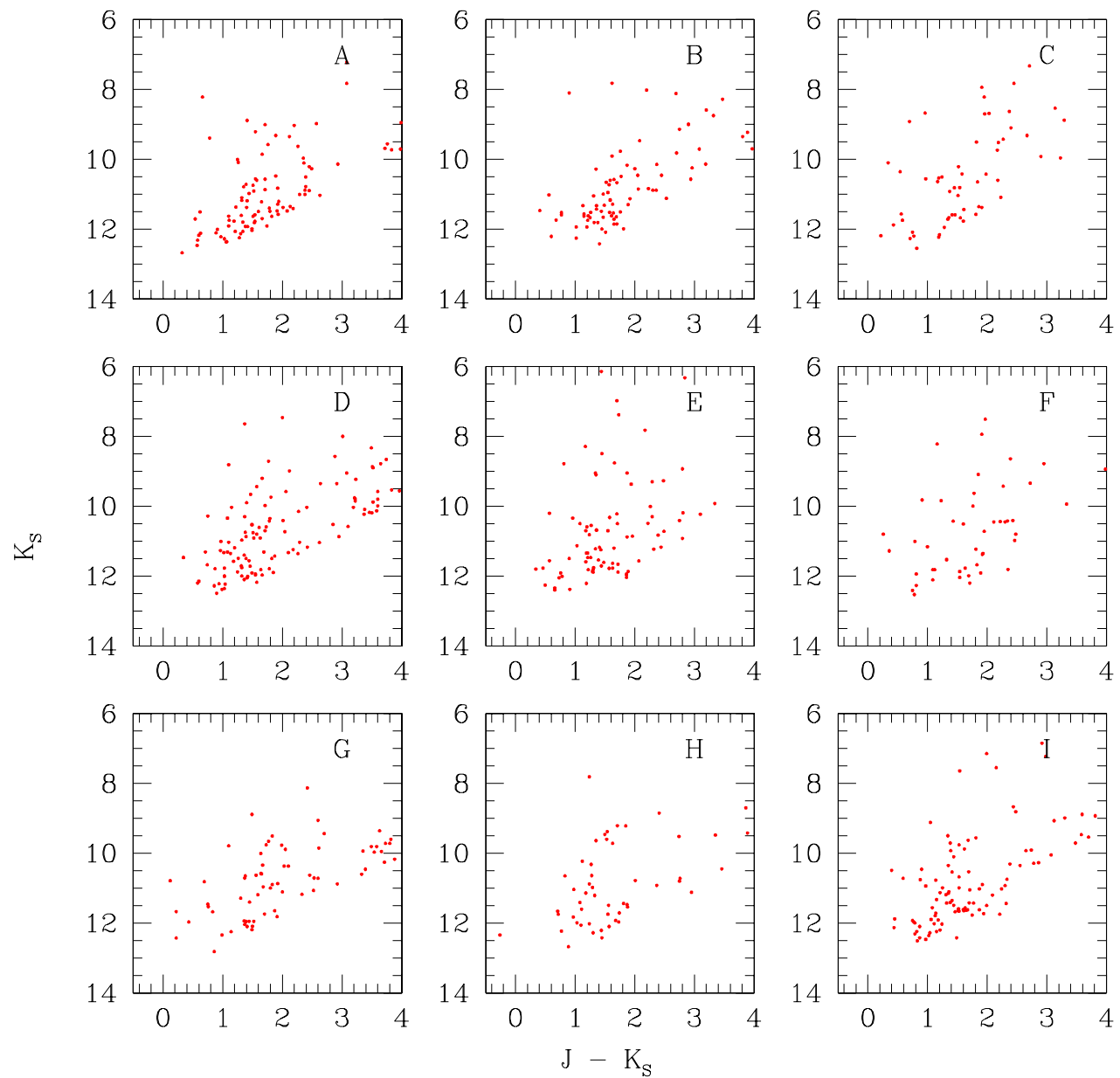

Figure 1. $K_{S}$ vs $J-K_{S}$ for 9 locations around $\mathrm{RA}=17: 48$ and $\mathrm{DEC}=-28: 20$. 

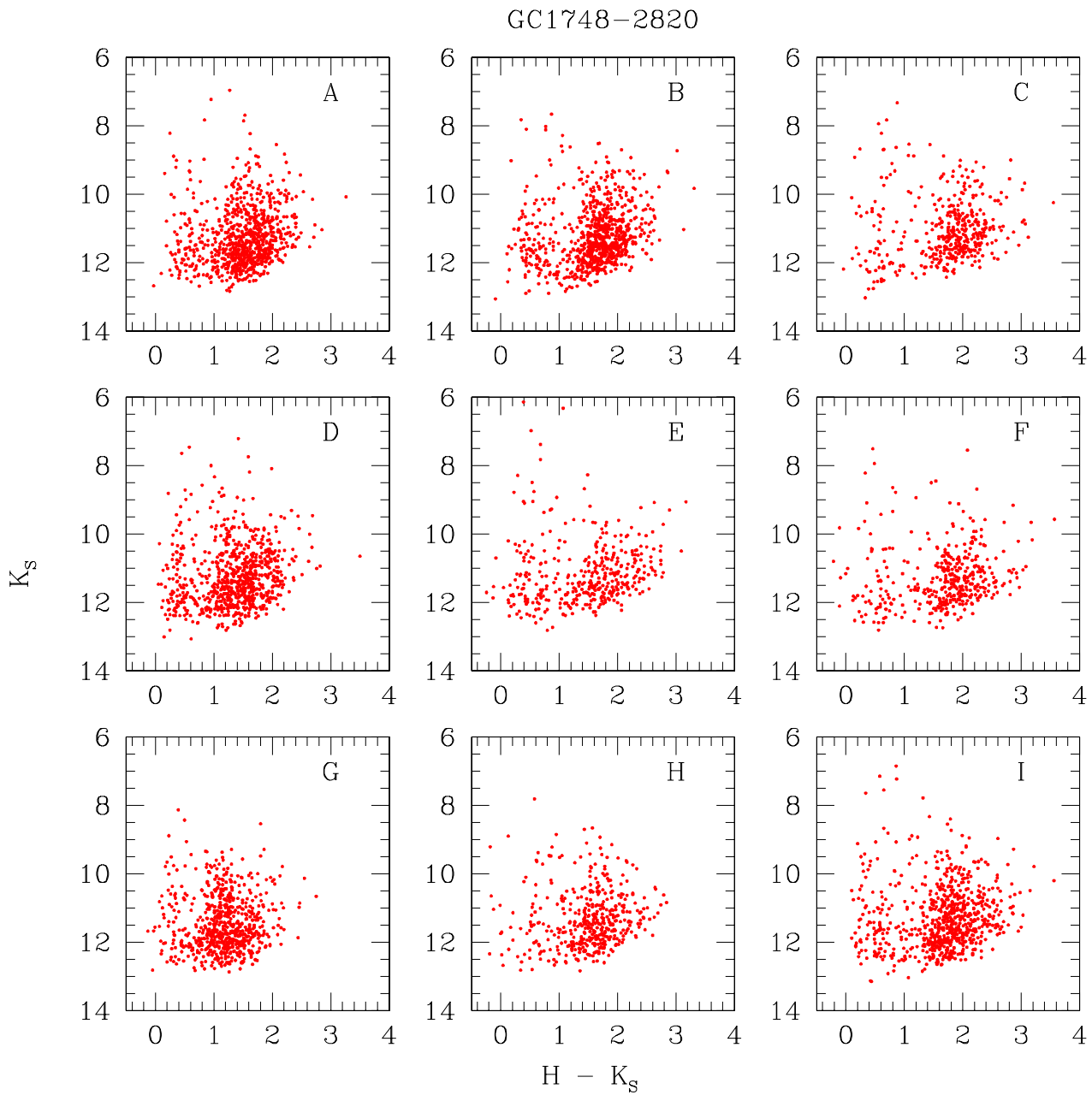

Figure 2. $K_{S}$ vs $H-K_{S}$ for 9 locations around $\mathrm{RA}=17: 48$ and $\mathrm{DEC}=-28: 20$. 


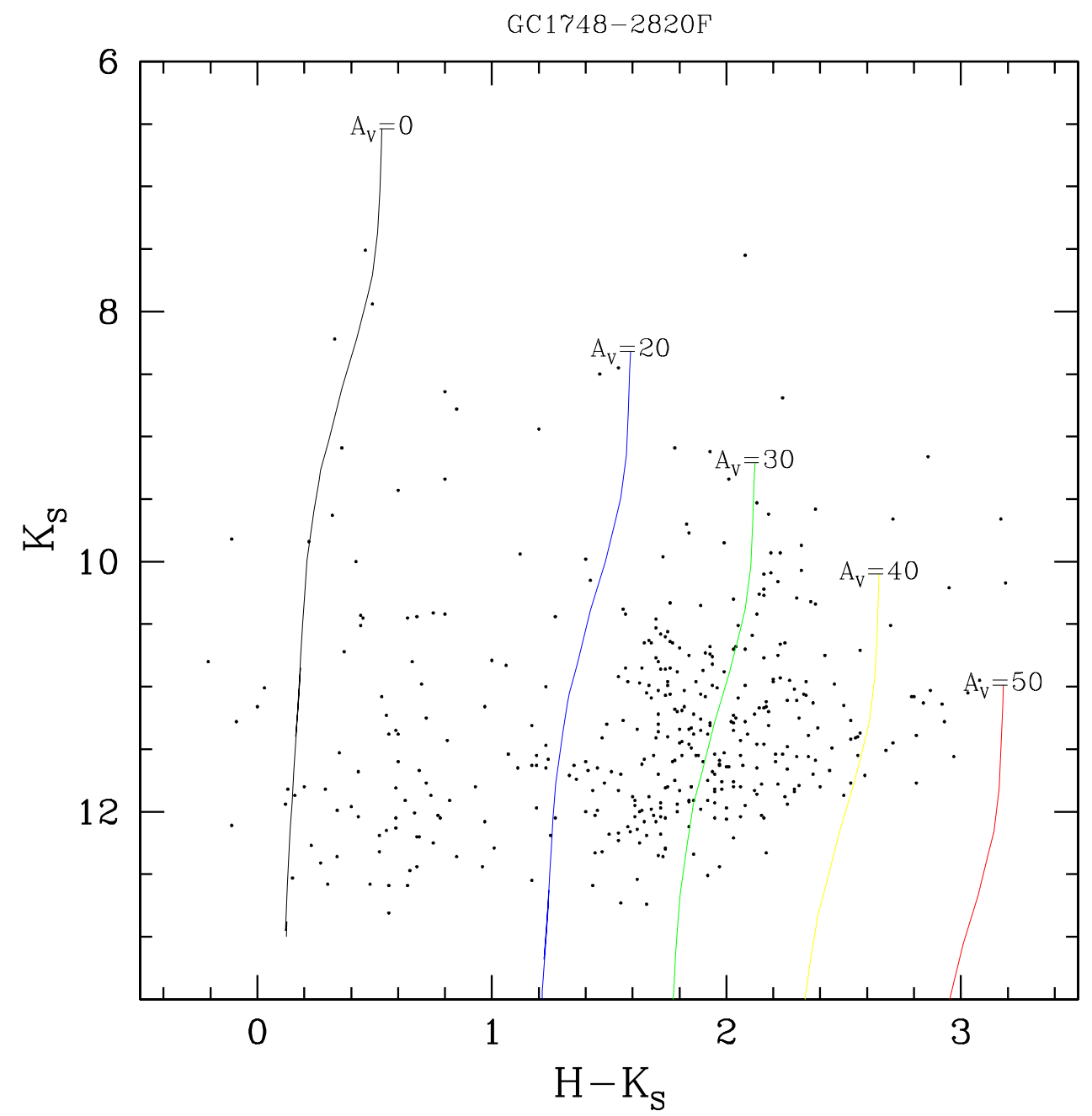

Figure 3. $K_{S}$ vs $H-K_{S}$ for one location towards $\mathrm{RA}=17: 48$ and $\mathrm{DEC}=-28: 20$ with red giant isochrones overplotted at various $A_{V}$. 